\title{
GRANDES NEVADAS Y PERCEPCIÓN DE LAS MISMAS EN ALCOY
}

\author{
Enrique Moltó Mantero*
}

\section{RESUMEN}

Las precipitaciones en forma de nieve no son un fenómeno extraordinario en la Hoya de Alcoy, aunque tampoco podamos considerarlas como algo absolutamente rutinario. En este sentido, podemos hablar de nevadas en los puntos más elevados de la comarca más de una vez al año, de precipitaciones débiles en forma de nieve que no llegan a blanquear las calles de los principales núcleos urbanos con presencia anual, $\mathrm{y}$, con frecuencia de repetición algo menor, de nevadas moderadas que dan espesores de entre 5 y 20 $\mathrm{cm}$. en casi todos los pueblos de este ámbito geográfico. Con intervalos mayores, que trataremos de determinar, la intensidad de las nevadas en esta área geográfica, donde se llegan a acumular más de cincuenta centímetros de espesor en pocas horas, puede traer funestas consecuencias para las infraestructuras y actividades económicas, esencialmente para la industria. En estos casos podemos hablar de la nieve como riesgo climático. Todas estas nevadas son percibidas de muy diversas maneras por los habitantes de esta comarca, y la prensa se muestra como una fuente de información especialmente útil para expresar estas percepciones.

Palabras clave: Hoya de Alcoy, nevadas copiosas, fenómeno recurrente, efectos económicos y territoriales, riesgo climático, percepción y prensa.

\section{ABSTRACT}

Snow precipitations are not an unusual phenomena in Alcoy Valley, but they are not either absolutely routinely. There are snowfalls over the highest land in the area almost once time on the year. There are weak snowfalls (annual recurrence) that do not cover the streets of the main towns and other ones with a lower recurrence, which reach depth between 5 and $20 \mathrm{~cm}$. Finally there are snowstorms with a variable recurrence, that have a remarkable vividness: they can accumulated a depth of $50 \mathrm{~cm}$ in a few hours. These cause terrible consequences on infrastructures and economic activities, particularly to the manufactures. In these last events, it is talk of snow as a climatic risk. These snowfalls

\footnotetext{
* Instituto Universitario de Geografía. Universidad de Alicante.
} 
are noticed in different ways by the inhabitants of this area. Newspapers are an expressive source of information to be stated these ideas.

Key words: Alcoy area, snow waves, recurrent phenomenon, economic and territorial consequences, climatic risk, perception and newspaper.

\section{Introducción}

Este artículo intenta resumir un trabajo de investigación más amplio sobre las peculiaridades de los temporales de nieve en la Hoya de Alcoy, que pretende recoger las configuraciones sinópticas que los originan, las características de los mismos, el tratamiento que les ha dado la prensa en los últimos 120 años, la percepción de las nevadas por parte de los distintos segmentos de la población y los impactos positivos y negativos en el medio y en las actividades económicas.

El guión a seguir en este artículo se iniciaría con una breve exposición de las causas atmosféricas de los temporales de nieve, posteriormente se hace una descripción genérica de las peculiaridades de las nevadas en la Hoya de Alcoy, con una esquemática tipología de las mismas y, finalmente, se relatan, esencialmente a través de informaciones periodísticas, y a modo de apunte, algunos de los temporales de nieve más destacados desde 1883 hasta 1994, entre los que descuellan los de diciembre de 1926 y de 1883 y, en menor medida, el de enero de 1980, haciendo especial incidencia en la ambivalente percepción de las nevadas por parte de la población local.

\section{Causas atmosféricas de las nevadas}

Los temporales de frío intenso y nieve se desarrollan, preferentemente, en los meses de noviembre a abril, con ápice en diciembre a febrero. Estos meses han conocido las olas de frío y nieve más intensas acaecidas en las tierras ibéricas en el presente siglo.

Aspecto esencial resulta la relación entre los valores térmicos de mínimo valor alcanzados en los diversos episodios de frío intenso y la presencia en altitud de una u otra configuración sinóptica asociadas a la presencia en altitud de masas de aire de naturaleza diversa. Aunque son más las situaciones sinópticas relacionadas con esas situaciones, nosotros hemos de centrar el interés sólo en aquéllas que suelen determinar la presencia de nevadas en cotas bajas, además de la única presencia de bajas temperaturas. En este sentido, analizaremos las ondas árticas con procesos de retrogresión que dan lugar a vaguadas del noreste.

Las ondas árticas con eje mediterráneo son las configuraciones, que han motivado el mayor número de episodios de helada de este grupo, se asocian a la presencia de una vaguada con eje situado entre 5 y $10^{\circ}$ este. Se trata de valles planetarios profundos dirigidos desde el origen de la expansión hacia el ámbito mediterráneo que extienden las condiciones de baja presión por el espacio sinóptico europeo y cuenca occidental mediterránea hasta el norte de África, y que se vinculan con la presencia de elevadas crestas subtropicales en el Atlántico oriental, con encimera a 9.000-9.120 m. en 300 Hpa y eje en torno a 10-15 oeste. En el seno de estas vaguadas árticas mediterráneas es común, en virtud de su escasa longitud de onda, la gestación de depresiones frías con valores térmicos entre -36 y $-28^{\circ} \mathrm{C}$ en $500 \mathrm{Hpa}$. Esta dinámica atmosférica de tipo mixto se refleja, en superficie, con la dis- 
posición de un campo de baja presión bajo la onda ártica de altitud, que se encuentra minimizado ante el desarrollo de potentísimos anticiclones de bloqueo atlánticos de disposición meridiana y ápice (1.032-1.036 HPa) ubicado al oeste de las Islas Británicas. Las condiciones de baroclinia atmosférica suelen favorecer la gestación, a lo largo del episodio, de «depresiones de Génova», desarrollo ciclogenético alpino común en la cuenca occidental mediterránea. A estos vórtices se suelen vincular superficies frontales de carácter frío activas cuyas colas llegan a barrer la mitad oriental peninsular. Esta borrasca de filiación mediterránea es, por tanto, reflejo evidente de la presencia de aire frío en altitud sobre el Mediterráneo Occidental.

La instalación de una vaguada de evolución retrógrada sobre el espacio sinóptico peninsular, en los meses invernales, se asocia a la presencia de aire polar continental que procede del este de Europa, si bien, en ocasiones, llega a conectar con la masa de aire generada en la propia llanura siberiana. En estas circunstancias, la disposición de los campos de presión en superficie favorece la gestación de regímenes de viento del primer cuadrante que ocasionan acusadísimos descensos de temperatura.

La instalación de esta masa de aire muy fría en la columna atmosférica se manifiesta con la aparición, en superficie, de desarrollos ciclogenéticos con su núcleo sobre las latitudes peninsulares. En otras ocasiones son borrascas atlánticas de estructura frontal que se cuelan al sur de la dorsal anticiclónica y penetran por el Golfo de Cádiz hacia el Mediterráneo. La aparición de estas estructuras de baja presión proporciona las condiciones idóneas para el desarrollo de precipitaciones de nieve. Un buen ejemplo de este tipo de configuraciones lo tenemos en el mapa de superficie del día 26 de diciembre de 1926 (Fig. 1) ${ }^{1}$.

\section{Características de las nevadas}

Resulta obligado apuntar una serie de peculiaridades de las nevadas en esta comarca, estrechamente relacionadas con la forma de precipitarse y con los impactos territoriales que de ello se derivan.

En primer lugar, conviene apuntar el número medio de días de precipitación en forma de nieve en algunos puntos de esta comarca era, según Kunow, de 4 en Agres, 3 en Alcoy y 2 en Cocentaina $^{2}$, en función de la altitud de cada uno de estos observatorios. Por otra parte, el tratamiento día a día de la serie 1976-84 arroja un resultado de 1,5 días al año en Alcoy ${ }^{3}$. Todos estos promedios son algo mayores en los puntos elevados de este espacio geográfico, a altitudes generalmente superiores a los 1.000 metros. Estos valores pueden resultar ridículos en comparación con los alcanzados en otros ámbitos montañosos de la Península, pero nos indican ya que la presencia de este hidrometeoro, al menos una vez al año, es relativamente «habitual» en esta comarca.

La simple lectura de estos promedios, como suele ocurrir con otros datos que hablan de medias en el estudio de los climas, y en especial en un clima tan irregular en su comportamiento como el que aquí se da, no dice gran cosa de las características de las nevadas en esta comarca. Para ello hay que descender al estudio más detallado de los distintos casos conocidos, que podemos sistematizar con la elaboración de una tipología básica de las nevadas en este ámbito geográfico. Esta tipología se elabora en función de la cantidad de

1 OLCINA CANTOS, J. y MOLTÓ MANTERO, E. (1999): «La nevada de 1926. Repercusiones en la Montaña Alcoyana (Alicante)», Nimbus, n 3, Universidad de Almería, pp. 105-137.

2 KUNOW, P. (1966): El Clima de Valencia y Baleares, Valencia, Instituto Alfonso el Magnánimo, 239 pp.

3 GUALDA GÓMEZ, C.E. (1988): La Sierra de Mariola, Alicante, Universidad de Alicante, 268 pp. 


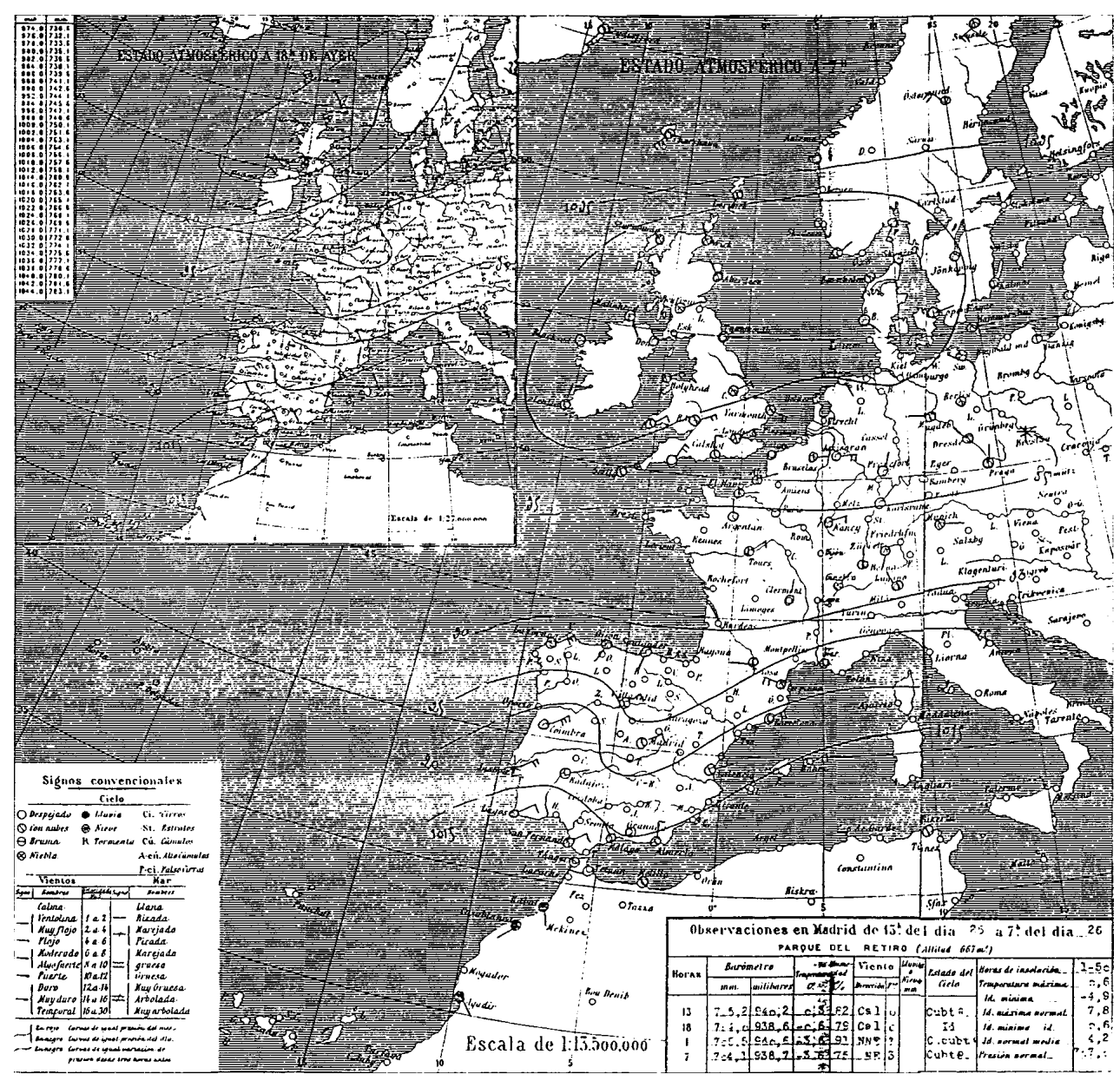

FIGURA 1. Situación atmosférica del 26 de diciembre de 1926. Boletín Meteorológico del Servicio Meteorológico Español. Cedida por Jorge Olcina Cantos.

nieve precipitada y la intensidad horaria de la misma, de las temperaturas que las acompañan, de los efectos sobre el medio, las comunicaciones y las actividades económicas, y de las distintas percepciones que se tiene de cada una de ellas.

a) Nevadas copiosas en los relieves circundantes por encima de los 600-700 metros, que son precipitaciones de lluvia o aguanieve sobre el núcleo urbano de Alcoy sin llegar a cuajar en las calles. Estas nevadas, cuando se producen en fechas plenamente invernales, suelen ser más beneficiosas que perjudiciales, en especial para la recarga de los acuíferos, y sólo pueden crear problemas en las comunicaciones al bloquear algunos de los puertos de montaña que dan acceso a la ciudad de Alcoy, y en los municipios rurales situados a mayor altitud. Un ejemplo reciente de este tipo de nevadas lo tenemos el 5 de diciembre de 1997, donde se recogieron $70 \mathrm{~mm}$. en 24 horas en forma de agua o agua- 
nieve en la ciudad de Alcoy, que fueron mayoritariamente en forma de nieve por encima de los 700 metros.

Estas nevadas, según se deduce de distintas entrevistas orales y de referencias en prensa, no son percibidas como tales por los alcoyanos porque no cuajan en el núcleo urbano de la ciudad, no suponen un cambio brusco del paisaje urbano, aunque la nieve pueda ser vista desde la ciudad en los relieves que la circundan. Generan un cierto tipo de frustración entre aquéllos que esperaban la nevada en el propio núcleo urbano para poder xafar neu, que es en cierto modo compensada con el desplazamiento, en ocasiones casi masivo, especialmente durante el fin de semana siguiente a la nevada, no sólo de los alcoyanos sino de algunos habitantes del litoral próximo, hacia algunas de las montañas que son capaces, según su exposición y las condiciones atmosféricas reinantes, de mantener durante más tiempo el manto blanco. Como muestra de la percepción de estas nevadas tenemos el siguiente comentario de prensa: «...entendiendo como tal que la nieve llegue a cuajar totalmente en las calles de la población. Una nevada que blanquee la Font Roja o el Montcabrer no es una nevada, es una anécdota.» ${ }^{4}$

En fechas actuales no son pocos los que achacan al «cambio climático» el hecho de que estas nevadas cuajen en altitudes ligeramente superiores pero no sean capaces de hacerlo en el núcleo urbano. Sin olvidar la posible influencia del efecto real de la «isla de calor urbano» que dificultaría la caída de nieve y el hecho de que la misma cuajara en las calles cuando estos temporales van acompañados de una importante carga de humedad y las temperaturas oscilan entre $0^{\circ} \mathrm{C}$ y $1^{\circ} \mathrm{C}$, cabe recordar que el 13 de enero de 1980 , fecha en la que las temperaturas apenas bajaron de $\operatorname{los} 0^{\circ} \mathrm{C}$ y el efecto anteriormente mencionado ya sería considerable, la nevada fue de gran envergadura porque sin duda se dieron las condiciones atmosféricas idóneas para que esta se produjera, obviando cualquier interferencia de la actividad humana. Igualmente, cuando las nevadas tienen lugar con temperaturas claramente inferiores a $\operatorname{los} 0^{\circ} \mathrm{C}$, como el 18 de enero de 1994, y las condiciones de humedad son las idóneas ésta se produce. Ello no quiere decir que la actividad antrópica no incida, nunca con exclusividad, por ejemplo en el hecho de que la nieve tenga más facilidad para cuajar durante la noche o la madrugada que durante el día por razones obvias, pero siempre estamos hablando de casos en los que las condiciones atmosféricas reinantes están en el límite de la idoneidad para que se produzca una nevada, porque cuando éstas son muy favorables todos estos efectos son minorados.

b) Precipitaciones poco cuantiosas, 1 a 5 mm., originadas por depresiones de escasa entidad y en conjunción con un aire muy frío y seco, con vientos generalmente del primer cuadrante, pero también del cuarto, que, debido a la presencia de temperaturas muy bajas, caen en forma de nieve por encima de los 400 metros y afectan a casi toda la Hoya, pero que son poco menos que anecdóticas y apenas llegan a cuajar en algunos tejados y campos, y no ocasionan ningún tipo de problemas, aunque tampoco muchos beneficios. Los únicos problemas pueden venir dados por las bajas temperaturas alcanzadas, especialmente por las heladas en algunos cultivos, si estos episodios tienen lugar en fechas casi primaverales. Son junto con los primeros, los episodios más frecuentes, repetidos al menos una vez casi todos los años.

La percepción que se tiene de las mismas es similar a las del grupo anterior. Aunque desde el estricto punto de vista de la estadística de la observación meteorológica puedan ser

4 Ciudad de Alcoy, 19 de enero de 1994. 
contabilizadas como precipitación en forma de nieve, no son vividas como nevadas por los habitantes de la ciudad, por las mismas razones apuntadas para el caso anterior. Aunque, a diferencia de las anteriores, sí se vean ligeras capas de nieve en los tejados, coches y jardines en el propio núcleo urbano, son consideradas como nevadas a medias, «incapaces de cumplir con el objetivo de blanquear por completo las calles de la ciudad», y que apenas persisten sobre las superficies en donde cuajaron ante los primeros rayos de sol. Aunque desde un punto de vista estrictamente objetivo sean mucho menos beneficiosas que las apuntadas con anterioridad por la escasa aportación hídrica que llevan aparejada, suelen ser valoradas positivamente desde el punto de vista de la percepción subjetiva de los habitantes de la ciudad como «purificadoras del ambiente».

c) Precipitaciones moderadas, entre 10 y $30 \mathrm{~mm}$. en 24 horas, que acumulan espesores variables en función de la temperatura alcanzada, que determina el carácter más o menos sólido del hidrometeoro, y la forma más o menos intensa de producirse, que oscilan entre 10 y los 25 centímetros en las propias calles de la ciudad de Alcoy, y que pueden alcanzar mayor volumen en los relieves y áreas rurales circundantes. Son percibidas por la población como auténticas nevadas y originan ya cierto tipo de problemas, especialmente en las comunicaciones con bloqueos más o menos intensos y duraderos no ya con el exterior de la Hoya, sino dentro de los propios núcleos urbanos. No suelen producirse daños de consideración en las infraestructuras y en los edificios, y si a ello unimos el hecho de que se produzcan en fechas plenamente invernales, el balance del impacto de estas nevadas, especialmente para la agricultura y la recarga de acuíferos es claramente positivo, a pesar de los problemas generados, básicamente en las comunicaciones, por lo que en las distintas referencias encontradas en la prensa la valoración es casi siempre claramente positiva hasta el extremo de hablar de la nevada como auténtica fiesta ${ }^{5}$. En los últimos 19 años podemos citar como ejemplos de este tipo de nevadas los de 11 de enero de 1981, 23 de marzo de 1982, 12 de febrero de 1983, 14 de febrero de 1984, 24 de enero de 1992, 28 de febrero de 1993 y 18 de enero de1994, y ello nos daría un periodo de repetición de 2,7 años, que oculta, como se observa leyendo las fechas anteriores, una gran irregularidad en la repetición del fenómeno, difícil de medir desde un punto de vista estadístico. Son nevadas que desde el punto de vista del desbloqueo de las vías de comunicación y la solución de determinados casos urgentes ya exigen cierta previsión en las políticas de planeamiento y ordenación del territorio.

d) Las nevadas más destacables por el impacto que tienen sobre el territorio son aquéllas muy intensas, que acumulan alrededor de $50 \mathrm{~cm}$. de nieve en periodos que oscilan entre las 24 y las 72 horas, en los propios núcleos urbanos de los municipios más poblados e industrializados de la comarca. No hablamos aquí de altura de precipitación por varias razones: en primer lugar porque lo verdaderamente determinante a la hora de medir los impactos de estas nevadas en comunicaciones, infraestructuras y edificios, es el dato del espesor alcanzado; en segundo lugar porque en el caso de las nevadas anteriores a 1960 no disponemos de datos fiables sobre volúmenes de precipitación; también se ha de tener en cuenta que no es fácil encontrar una equivalencia precisa entre litros de agua precipitados y espesor de nieve alcanzado ya que éste depende de múltiples factores, esencialmente

5 El titular del Ciudad de Alcoy del 19 de enero de 1994 no puede ser más ilustrativo: «La nevada fue una fiesta». 


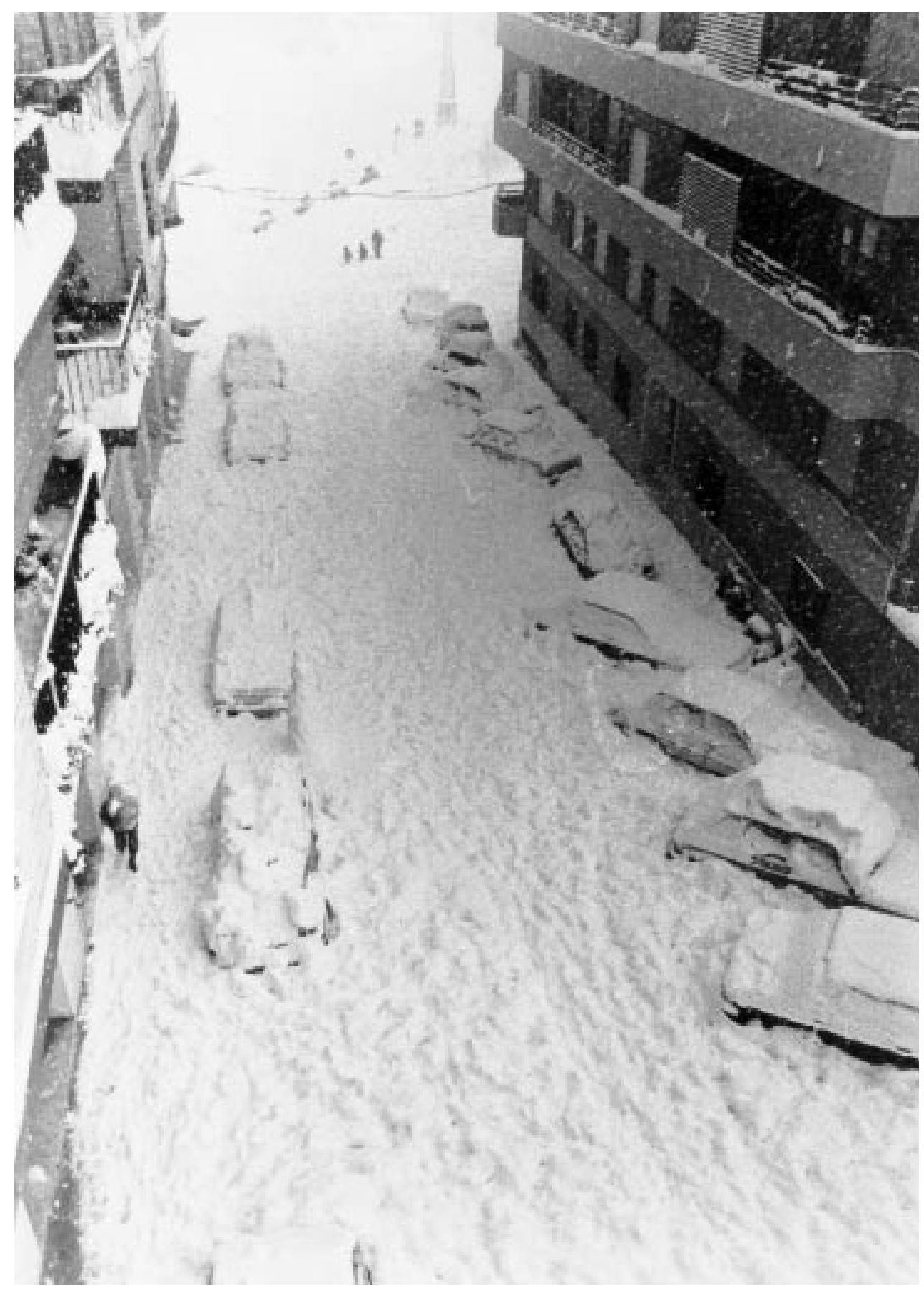

FIGURA 2. Foto realizada durante la nevada del 13 de enero de 1980 por Paco Grau en la que se observa el espesor alcanzado en las calles de Alcoy por la nieve. 
humedad, temperatura y orientación respecto al viento que conduce la nevada, y la misma cantidad de litros/metro cuadrado puede dar distintos volúmenes de nieve, en distintos momentos y en diferentes lugares en el mismo temporal. Un ejemplo de este último aspecto lo podemos tener en el hecho de que la cantidad de precipitación en los episodios de 1926 y 1980 pudo ser similar y dio espesores dispares, sin duda por el hecho de que de que en el primer temporal las temperaturas fueron mucho más bajas y en el segundo apenas bajaron de los $0^{\circ} \mathrm{C}$ y por la propia influencia de la «isla de calor urbana» ya notable en 1980 . El umbral numérico elegido responde al hecho de que desde 1883 hasta 1999 se ha comprobado que las nevadas que se han acercado o han superado esos $50 \mathrm{~cm}$. han ocasionado ya graves daños en las comunicaciones, edificios, cultivos, industrias, etc., e incluso pérdida de alguna vida humana.

Los beneficios de estas copiosas nevadas son indiscutibles para la recarga de los acuíferos y en la mayoría de los cultivos, a pesar de los posibles destrozos en algunos árboles, pero no suelen compensar todo el daño producido en otras actividades económicas, esencialmente en la industria, actividad predominante en el intervalo de años analizado en los municipios más poblados, especialmente en Alcoy. No obstante, no dejan de encontrarse valoraciones positivas al espectáculo ofrecido por este tipo de nevadas y un cierto anhelo de que se produzcan como señales del fin de grandes sequías y saneadoras del ambiente. Los ejemplos más destacados en el periodo analizado son el del 8/XII/1883, enero de 1914, Navidad de 1926, 8/II/1942, 11/I/1960 y 13/I/1980, y ello nos da una frecuencia de aparición de 19,3 años para este tipo de grandes nevadas, sin olvidar la irregularidad ya mencionada, que hace que los años transcurridos entre este tipo de nevadas vayan desde los 12 hasta los 31. Son estas las nevadas que podemos considerar como riesgo climático dados los daños ocasionados, con un periodo de retorno variable pero seguro, que ha de ser tenido en cuenta en la planificación, para prevenir, en la medida de lo posible, sus efectos, ya que estamos hablando de un fenómeno meteorológico no rutinario, pero en modo alguno excepcional. La actitud ante este tipo de sucesos debería ser en cierto modo comparable a la mantenida ante las precipitaciones de agua de fuerte intensidad horaria que ocasionan extraordinarias avenidas fluviales, especialmente en el ámbito geográfico mediterráneo, que tienen periodos de retorno variables, pero cuya presencia está asegurada por las características del clima mediterráneo.

Una peculiaridad de estas nevadas que puede distinguirlas de las que tienen lugar en otros lugares de la Península más que los espesores alcanzados, es el hecho de que se acumulan en muy pocas horas, característica paradigmática de las precipitaciones en el clima mediterráneo. Como ejemplos de esta intensidad en 1926 el metro de espesor se alcanzó en tres días, pero especialmente se concentró en las 24 horas del día 26, y en 1980110 $\mathrm{mm}$. precipitados en forma de nieve más 50 en forma líquida, que dieron un espesor máximo en torno a los $60 \mathrm{~cm}$. en el núcleo urbano de Alcoy, cayeron en tan sólo 30 horas (Fig. 2).

\section{Grandes nevadas en la montaña alcoyana entre 1883 y 1999}

Para este período se han hallado seis nevadas que se acercan o superan con creces 50 $\mathrm{cm}$. de espesor, que ocasionan daños importantes, y podemos considerarlas como riesgos climáticos. La información disponible sobre las mismas, esencialmente prensa escrita, no es uniforme, destacando especialmente en cantidad y calidad la referida a la Navidad de 1926 y la del 13 de enero de 1980, siendo muy escasa y exclusivamente oral, la de 1942. 
Todas ellas, especialmente las de 1883, 1926 y 1980, son consideradas además como hitos en la historia de Alcoy en el último siglo.

\subsection{Aspectos cuantitativos de los temporales}

Parece conveniente iniciar este apartado con la nevada que es punto de referencia de todas las demás, permanentemente evocada cada vez que se produce una nevada mayor o menor sobre Alcoy, la gran nevada de 1926. A mediados de la década de los veinte tiene lugar una de las más intensas olas de frío y nieve ocurridas en tierras peninsulares a lo largo del siglo XX. Esta nevada se ha convertido en Alcoy en un punto de referencia histórico para sus habitantes. La nevada de las navidades de 1926 batió, al menos en este siglo, todos los registros. A la hora de hablar de espesores alcanzados es obligado citar la medida que figura en la placa de la ermita de la Virgen de los Lirios, en el Santuario de la Font Roja, a una altitud próxima a los 1.100 metros, donde reza con carácter conmemorativo la siguiente leyenda «Hasta aquí llegó la nieve. 27 de diciembre de 1926. 2,10 metros.» (Fig. 3) En el mismo sentido, en una sección de La Gaceta del día 29/XII/1926 se indica que: «una copiosa nevada, que si al principio no se manifestó violenta, ha sido algunas horas imponente y amenazadora. En grandes y compactos copos ha caído la nieve sobre nuestra población y sus alrededores, llegando su nivel, en muchos lugares a sobrepasar de un metro.»

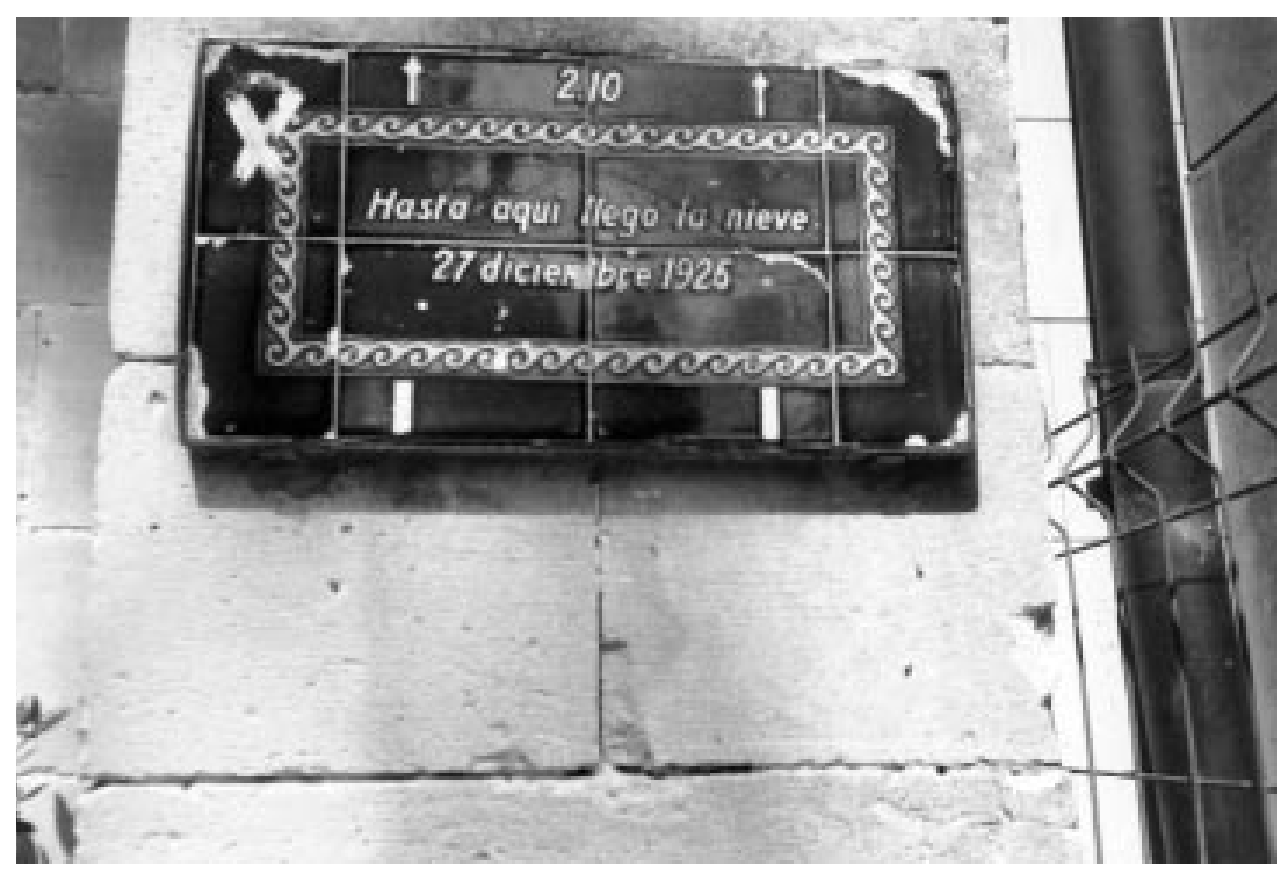

FIgURA 3. Detalle de la placa instalada en el Santuario de la Virgen de los Lirios (Parque natural de la Font Roja), con indicación de la altura alzanzada por la nieve en el temporal de las navidades de 1926. 
Muy interesante resulta la información referida al estado de las calles en los días posteriores a la nevada, ya que en ellas se comprueba la permanencia de la misma en las calles y tejados. Según algunas referencias orales, la nieve extraída para abrir esas brechas quedaba a ambos lados y, unida a la precipitada y a la despejada de los tejados, daba un espesor aún más imponente, que hacía que en algunas casas se entrara por el primer piso (Fig. 4). Diecisiete días después sigue más o menos presente la nieve en el núcleo urbano como se deduce de la lectura en La Gaceta de Levante de una cita referida a la presencia de la nieve en las calles, del día 12 de enero de 1927.

La nevada de 8 de diciembre de 1883, evocada en La Gaceta en 1926 como de mayor espesor aún, no parece que distara mucho de ésta, e incluso puede que fuese bastante menor, por las informaciones aparecidas en el diario El Serpis el 10 de diciembre de 1883, que en ningún caso hablan de un fenómeno extraordinario ni indican espesor de referencia alguno, aunque si señalan una permanencia de la nieve en las calles superior a los siete días, resaltando más la molestia del barro que de la nieve, aunque ello pudiera deberse al hecho de que a esta nevada le siguiera una situación atmosférica más propicia a la disolución de la nieve que a la de 1926.

Las nevadas de enero de 1914 y 1960 llegaron a los cincuenta centímetros, como se deduce de las siguientes informaciones aparecidas en la prensa de aquellos años: «El pasado viernes cayó sobre nuestra ciudad una fuerte nevada, alcanzando el frígido elemento un espesor de cincuenta centímetros» 6 . «En términos generales la nieve alcanzó una altura de cincuenta centímetros ${ }^{7}$. La de 1980 pudo llegar a superar el medio metro en algunos puntos: «El total de nieve registrado en nuestra ciudad estos días, ha oscilado, según zonas, entre los 40 y los 60 centímetros de altura. ${ }^{8}$ No hay suficientes datos escri-

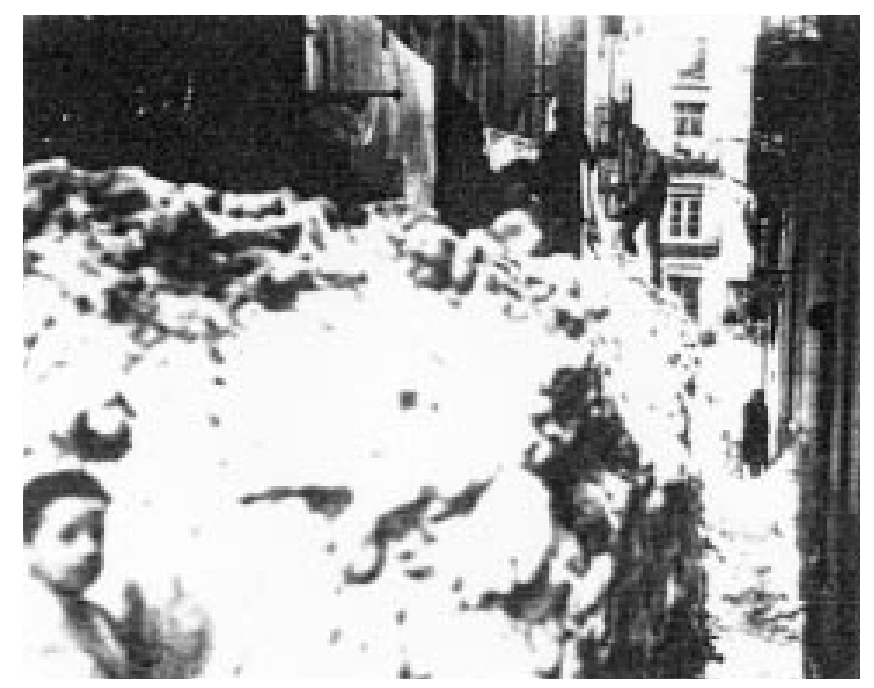

FIgURA 4. Escenas urbanas de Alcoy tras la nevada de 1926. Se aprecia bien la altura alcanzada por la nieve (más de 2 metros) en algunas calles. Foto facilitada por Paco Grau.

6 Heraldo de Alcoy, 8 de enero de 1914.

7 Ciudad de Alcoy, 19 de enero de 1960.

8 Ibidem, 15 de enero de 1980. 
tos sobre la nevada del 8 de febrero de 1942, la más destacada de las varias importantes que tuvieron lugar en los cuarenta, pero, según referencias orales, aunque quedara bastante lejos de esos 50 centímetros en el núcleo urbano de Alcoy, pudo superarlos en áreas rurales próximas con exposición favorable y fue persistente en las calles por la situación atmosférica que la siguió y por los pocos medios disponibles en plena posguerra para despejarla.

\subsection{Efectos en las infraestructuras urbanas y comunicaciones}

Uno de los efectos más destacados de las nevadas en este área geográfica es el del bloqueo en las comunicaciones, para el que ni siquiera hace falta que tenga lugar uno de estos grandes temporales.

El colapso urbano sufrido en la ciudad a causa de la nieve el día 26 de diciembre de 1926 se relata con profusión en las crónicas de la Gaceta del día 29 de diciembre de 1926. Así se indica, por ejemplo que: «los servicios públicos interrumpidos... Por consecuencia las calles, aunque ofrecían un pintoresco aspecto, se han hecho intransitables, hasta el extremo de hallarse obstruidas totalmente. Los servicios públicos de Telégrafos, Teléfono, Electricidad y el de trenes han sufrido importantes averías en sus líneas quedando suspendidos, hasta que, cesada la tormenta y reparados los daños considerables, puedan otra vez funcionar normalmente... Muchos cables eléctricos y telefónicos se encuentran rotos, hallándose, por tal motivo, la población aislada, y sin el servicio de alumbrado público y particular.»

En esta nevada el aislamiento por ferrocarril con la localidad de Gandía se prolongó hasta el 30 de diciembre, un tiempo relativamente corto, si tenemos en cuenta que nos encontramos a comienzos de siglo. El aislamiento con Játiva por ferrocarril fue mayor por las dificultades de despejar la nieve de la vía al transcurrir ésta a mayor altitud y por relieves más abruptos ${ }^{9}$.

En su conjunto es necesario resaltar la importancia del transporte ferroviario, a pesar de la existencia ya de carreteras, utilizadas por carros y algunos coches. El bloqueo de éstas fue mayor ante los escasos medios disponibles para despejarlas, a diferencia de lo que permitía el uso de máquinas exploradoras para quitar la nieve de los raíles en el caso del ferrocarril (Fig. 5). Así el 12 de enero de 1927, 17 días después de la nevada, en La Gaceta de Levante se alude lo siguiente: «Servicio normalizado. Ayer llegó hasta Alicante por Ibi, Castalla y Agost un auto y estando expedita esta carretera hoy se normalizará el servicio de autos.» La incomunicación trae consigo falta de abastecimientos, especialmente en siglos pasados y a principios de éste, aunque también parece que hubo algún tipo de problemas más o menos reconocidos en la nevada de 1960, hecho que se dará cada vez menos ante las mayores posibilidades técnicas de superar los bloqueos y las mejores condiciones de almacenamiento de alimentos de hogares y comercios, pero que sigue presente en el subconsciente de muchos habitantes de estas comarcas, especialmente los de más edad. Este hecho se deduce de referencias orales y de la lectura de algunos artículos de fechas bastante recientes, en las que se deja ver cierta psicosis un tanto absurda de desabastecimiento. Podemos citar el siguiente ejemplo: «Sin problemas de abastecimiento. Es casi inevitable, pero sin demasiada lógica, ante una situación como la creada durante estos últimos días, se suscita una psicosis de abastecimiento en los productos más elementales y básicos. $Y$,

9 La Gaceta de Levante, Diario Independiente de Alcoy, 31 de diciembre de 1926. 
precisamente esa psicosis, lo único que puede generar es un agravamiento de la situación, al acumular en casas particulares alimentos.»10.

En la nevada de 1883 también hay referencias en la prensa, en concreto en el diario El Serpis, a la incomunicación, en esta época sólo por carruaje, con el exterior de la Hoya, aunque no se indica con claridad cuánto duraría ésta, pero parece que mucho menos que la que ocasionaría la nevada de 1926: «Es decir, que estamos poco menos que incomunicados con el resto del mundo.» ${ }^{11}$.

En 1960 hubo un importante bloqueo de las comunicaciones viarias que obligó al rescate aéreo de los ocupantes de varios vehículos en los puertos que dan acceso a la ciudad y en 1980 el bloqueo terrestre duró unas veinte horas. La incomunicación impuesta por la nevada de 1942 se debió más a la falta de medios para luchar contra la misma que al volumen de nieve acumulado.

\subsection{Efectos en las actividades económicas}

Los efectos de la nevada de 1926 en las industrias de la ciudad fueron considerables porque la nevada se produce, además, en un momento de grave crisis industrial. Los destrozos ocasionados por la nieve en las industrias, aparentemente peor preparadas entonces y ahora que las viviendas para soportar el peso de la misma en los techos, agravaban una situación ya delicada. La descripción de los daños ocasionados en industrias, comercios y viviendas ocupa un considerable espacio en la prensa, expresando en reiteradas ocasiones el hecho asombroso de que no hubiera víctimas mortales, aunque según informaciones de la propia Gaceta, se habrían producido tres muertos en los alrededores. Como ejemplo significativo podemos citar este párrafo aparecido el 31 de diciembre de 1926 en la Gaceta de Levante: "Las fábricas paradas, constituyen un grave problema. La inactividad es la falta de pan. Pero hay que fijarse en las proporciones de la nevada, para en medio de tanto mal, sacar las consecuencias de que todo ha sido un bien. Han habido derrumbamientos numerosos; algunos de ellos con proporciones verdaderamente catastróficas. Pérdidas de muchos miles de duros; pero pérdidas materiales. Invade los límites de lo extraordinario, el hecho de que con tanto desastre peligrosísimo no hayan ocurrido desgracias».

La ruina económica que supone este desastre es digna de comparar con la producida en otros lugares por las avenidas fluviales. En la ciudad de Alcoy podemos contrastarla con la producida por la nevada de 1980, a pesar de ser menor en espesor alcanzado que la de 1926, con pérdida de al menos 500 empleos, daños evaluados en mil millones de pesetas y merecedora de la declaración de zona catastrófica ${ }^{12}$, o con las lluvias torrenciales de septiembre-octubre de 1986. Acerca de esto cabe resaltar la escasa preparación de buena parte de las infraestructuras urbanas y de las construcciones, esencialmente las industriales, para soportar grandes nevadas, a pesar de tratarse de un fenómeno poco frecuente, pero en absoluto insólito. La mayor parte de los daños ocasionados por la nevada de 1980 se produjeron por el hundimiento de los tejados de muchas industrias (Fig. 5), que se hacían y se siguen haciendo con materiales poco resistentes para ahorrar gastos, e incluso de algunos edificios de viviendas: «Respecto al peso resistido por los tejados de la ciu-

10 Ciudad de Alcoy, 15 de enero de 1980.

11 El Serpis, 11 de diciembre de 1883.

12 Ciudad de Alcoy, 17 y 26 de enero de 1980. 


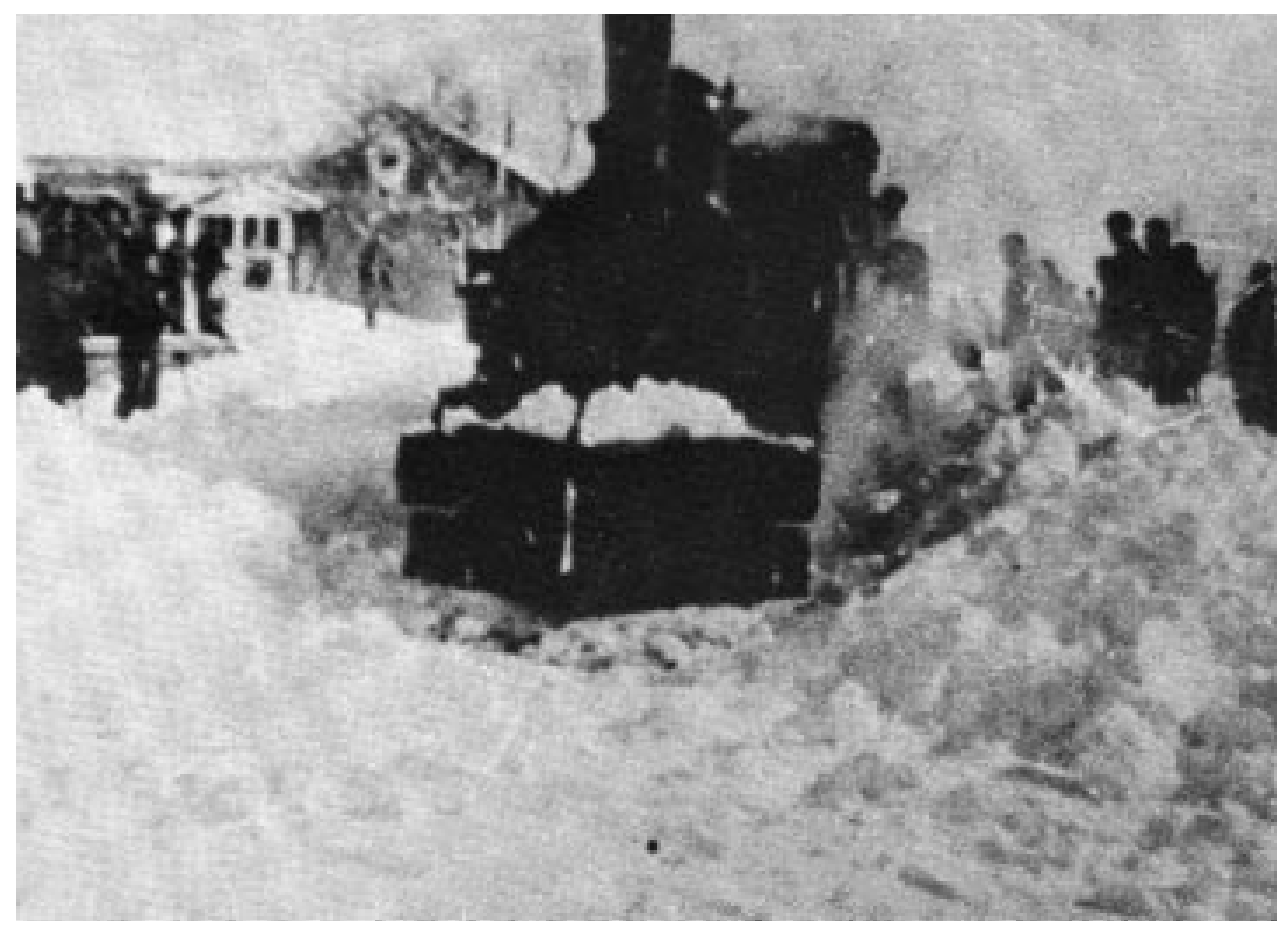

FIGURA 5. Máquina exploradora utilizada en la nevada de 1926 para despejar de nieve los raíles. Foto facilitada por Paco Grau.

dad, ha oscilado entre los 100 y 200 kilos por metro cuadrado. Hay que informar que las Ordenanzas municipales de nuestra ciudad obligan a un mínimo de resistencia en las construcciones de 80 kilos por metro cuadrado. Lo cual quiere decir que los hundimientos de techumbre hubieran podido ser mucho mayores. ${ }^{13}$. En las nevadas de 1883, 1914 y 1926 aparecen en los respectivos periódicos bandos municipales que ordenan que se libere cuanto antes de nieve a los tejados para evitar derrumbes, cosa que no sucede ya en las de 1960 y 1980, fechas estas últimas en las que teóricamente los edificios contarían con más resistencia, aunque cabe preguntarse si no se debe también esta diferencia a una mayor costumbre en aquellos años a desarrollar estas prácticas ante un mejor conocimiento por parte de los ciudadanos del medio natural que les envolvía, en el que son posibles cada cierto número de años estos temporales. En este sentido, creo que es necesario llevar a cabo una reflexión acerca de si hay una suficiente consideración de este tipo de riesgos climáticos en la planificación y ordenación del territorio de los municipios de estas comarcas.

13 Ibidem, 17 de enero de 1980

14 Acerca de la utilidad de la prensa como fuente de información para este tipo de eventos meteorológicos resulta de gran interés la lectura del libro coordinado por RUIZ DE URRESTARAZU, E. (1998): El clima del País Vasco a través de la prensa, Servicio Vasco de Meteorología, Grupo de Climatología de la Universidad del País Vasco, Vitoria, 212 pp. 


\subsection{Percepción de las nevadas en la montaña alcoyana}

Resulta muy ilustrativo la lectura de la serie de comentarios, publicados en la prensa local, que aluden a la percepción de las nevadas por parte de los alcoyanos ${ }^{14}$. Las informaciones y comentarios acerca de la nieve se convierten en una auténtica sección cada vez que se produce una nevada de cierta entidad en Alcoy, en la que se sigue un esquema similar, referido a la ciudad y a todos los municipios de la comarca: información cuantitativa sobre la nevada, medidas tomadas contra sus efectos, incluidas acciones de rescate, casi siempre valoradas positivamente, evaluación de los impactos en infraestructuras, comunicaciones y actividades económicas, evocación histórica de otras nevadas, especialmente de las más copiosas o más singulares, y valoración más o menos subjetiva de los efectos de la nevada. Por lo común estas opiniones manifiestan la doble faz que acompaña a los temporales de nieve, beneficiosos para el campo, la circulación superficial de aguas y la recarga de acuíferos, pero dañinos para el resto de actividades económicas, sobre todo por las pérdidas que supone la incomunicación momentánea y la rotura de infraestructuras e instalaciones.

Especialmente representativos de la percepción de las nevadas por parte de los alcoyanos resultan la larga serie de artículos de opinión recogidos por la Gaceta de Levante acerca de los efectos de la Gran Nevada de la Navidad de 1926. Así el día 29 de diciembre, en uno de estos artículos se expresa lo siguiente:

«Casi bate el récord el actual año de gracia, infinidad de postes, de instalaciones, claraboyas y tejados, con su aspecto doliente y desmayado, son testimonio de esto. Además el otro aspecto, un poco más serio, más trágico, industrias paralizadas, caseríos angustiosamente aislados, multitud de obreros sin pan; mucho frío, muchos miles de duros estérilmente perdidos. ¿Compensará todo esto de malo y de trágico el que los árboles se sacudan toda enfermedad y despierten lozanos, y que las tierras, fructifiquen luego, y que las fuentes afiancen su caudal? ¿Será esta nevada de evocación por los daños o por los beneficios? ¿O simplemente se recordará como a algo inusitado, avaro y codicioso de mostrarse dos veces a una generación? Dios haga que esta nevada se recuerde como a manantial de beneficios y como espectáculo caro, blanco y jubiloso.» ${ }^{15}$.

En sentido similar, dos días después se publica el siguiente artículo: «Comentario. Ya tenemos nieve para rato. La permanencia tenaz de ésta en los campos, después de todo, no es más que nuncio optimista de que la tierra expurgará toda maleza resurgiendo con ubérrimas frondosidades y cosechas felices y abundantes. En los caseríos, destrozos, derrumbamientos, escombros, ruinas, pérdida incalculable de riqueza. En los campos, simiente riquísima de valor incalculable que hará brotar manantiales por doquier y aplacará la sed del terruño en los ardorosos días estivales. Látigo y castigo por una parte. De otra, esta ruinosa plaga transformada en beneficios y fuentes de abundancia...Y ya no es solamente el peligro que han corrido los vecinos y los trabajadores de las casas y fábricas derrumbadas. El peligro ha existido en todos los trabajos que ha determinado las exigencias de las medidas tomadas para aligerar techumbres y aminorar molestias e incomodidades. En la descarga de terrazas y techumbres ha existido verdadero peligro. Los operarios realizaban su labor con una falta de precaución a todas luces reprobable. Se trabajaba con la risa en los labios y con un jolgorio de bodas; con estado de ánimo demasiado bullanguero y exento de la gravedad de peligro y de las circunstancias. Los paletazos, caían sin orden ni concierto, con manifiesto peligro de la seguridad del transeúnte. El habernos librado de

15 La Gaceta de Levante. Diario Independiente de Alcoy, 29 de diciembre de 1926. 
semejante bombardeo sin la rotura de alguna clavícula, ya es hecho que hace reflexionar y levantar el ánimo con gratitud. Realmente hemos pasado infinidad de molestias, disgustos por las considerables pérdidas materiales y por los perjuicios que supone la incomunicación con el resto de España; pero si se medita que no ha habido desgracias personales, pronto se encuentra la compensación de tanta pérdida. Se han perdido miles de duros; pero viven los damnificados que con tenacidad y empeño volverán a levantar sus edificios y a normalizar la vida. Conservemos lo que más vale: espíritu productor.» ${ }^{16}$.

Reflexión bastante medida con los pros y contras de la nevada en según que campos de la vida y la economía. Incluso cuando se hace referencia a los perjuicios hay una mirada optimista, que hace alusión a la falta de víctimas mortales y a la confianza en el «espíritu productor» de los alcoyanos. Anecdótica, pero significativa, resulta la crítica al jolgorio con que se tomaron las tareas del despeje de nieve de los tejados. La mezcla de goce y aflicción ante tan extraordinaria situación está siempre presente.

Resulta muy ilustrativo recoger la opinión expresada por el alcalde de Alcoy con motivo de otra nevada intensa, pero de corta duración, que dejo $18 \mathrm{~mm}$. el 28 de febrero de 1993, en poco más de tres horas. A consecuencia de este episodio la máxima autoridad municipal señaló lo siguiente: «su satisfacción por el comportamiento general de los ciudadanos ante la nevada sufrida por la ciudad en la noche del sábado y parte del domingo, confesando que: «la gente se ha portado responsablemente. No hemos de dramatizar el hecho de la nevada, que no ha generado por el momento ningún problema grave, y por otra parte, para los acuíferos y la salud pública, la nevada ha sido una bendición.» ${ }^{17}$ Llama la atención el hecho de que se haga este comentario después de uno de los meses de febrero más lluviosos del siglo en Alcoy, donde se recogieron más de 200 $\mathrm{mm}$. en 15 días, que ya habían dejado repletos, incluso saturados, los acuíferos, en los que esos 18 litros por metro cuadrado de la nevada no supondrían absolutamente nada. Esta opinión, singular y poco acertada, no deja de ser representativa de la percepción de las nevadas como indicadoras del fin de las sequías, como lo son en esta comarca y más aún en otros puntos del litoral las lluvias torrenciales, y como beneficiosas para la salud pública. Otro buen ejemplo de la asociación entre año seco desde un punto de vista pluviométrico y año sin nevadas de consideración en la mentalidad de los habitantes de estas comarcas lo tenemos en el siguiente comentario periodístico: «Y en el 79 no se registró ninguna nevada porque fue un año muy seco, con 271,5 litros de lluvia en los doce meses» ${ }^{18}$. Esta asociación no tiene sentido desde un punto de vista objetivo, porque años con totales pluviométricos inferiores a la media (490 mm.) como 1981 con $219 \mathrm{~mm}$. o 1983 con $267,5 \mathrm{~mm}$ sí registraron una nevada moderada y en años con elevada pluviometría como 1986 con 809,5 mm. o 1989 con 751, no se registró ninguna nevada digna de mención, pero es indicativa de la percepción de las nevadas como solución de las sequías.

Poco ha cambiado en la valoración de las nevadas por parte de los alcoyanos. Un hecho habitual cada vez que se produce una nevada de cierta intensidad sobre la ciudad es la evocación a la gran nevada de 1926, la nevada por excelencia. Este hecho resultó muy patente con motivo de la que se puede considerar segunda nevada del siglo en la montaña alicantina, ocurrida en enero de 1980: «Los más viejos del lugar sólo comparan este suceso cli-

16 Ibidem, 31 de diciembre de 1926.

17 Ciudad de Alcoy, 1 de marzo de 1993.

18 Ibidem, 19 de enero de 1994. 
matológico con el ocurrido - ya casi histórico- en el año 26... Comenzó el sábado sobre las once y con breves intermitencias no dejó de caer en casi treinta horas. Con diferencia de la del año 1926, que cayó toda en una sola noche.» ${ }^{19}$

Podríamos pensar que si, como se percibe en la actualidad, este tipo de nevadas tan intensas eran antes algo habitual, no deberían encontrarse evocaciones a nevadas anteriores con ocasión del temporal de frío y nieve de las navidades de 1926. Empero resulta curioso advertir que algún articulista hace mención a lo extraordinario de estos episodios, no recordados durante muchos lustros en la ciudad alcoyana:

«Nevada evocadora. Hay fechas, que tienen la virtud de pasar a la posteridad. Lo que tantos hombres se afanan por conseguir, y a cuyo fin dedican interminables noches de insomnios, inquietudes, trabajos, vigilias y sinsabores copiosos, sin resultado alguno casi siempre, lo consigue y de manera amplia e indeleble un día mimado por la temperatura glacial.

¿Quién no recuerda la famosa nevada de la Purísima? ¿Quién ignora los detalles de esta terrible nevada, transmitidos de padres a hijos? Aquella devastadora caída de nieve, marcó una fecha en el pueblo alcoyano, que sirve de referencia y cotejo, hasta para precisar años transcurridos y multitud de menudencias de la vida íntima. Son fechas éstas, de carácter inusitado, trascendental, dables solamente a los seres de una generación. Son espectáculos, caros, regios imponderables; tacaños y egoístas hasta el límite de no repetirse en media centuria. Se recuerdan tanto estos desbordamientos de la Naturaleza, porque no se prodigan. No es por el daño ni por los beneficios - que las dos cosas se danque pueda acarrear una riada arrasadora ni una nevada imponente, por lo que se recuerdan estas fechas memorables que se transmiten con la misma fidelidad que si se tratase de un acontecimiento militar o político de los que hacen ganar o perder un imperio.

Se recuerdan sencillamente, porque es espectáculo codicioso, avaro de repetirse, como si supiera que todo lo periódico pierde encanto y gana vulgaridad. Desde hace 44 años, hasta la fecha, no ha nevado con la cantidad suficiente, para que se pueda mentar la nevada de la Purísima, sin notorio agravio a la grandiosidad de ésta. Únicamente en el año catorce, hubo conato, asomo de nevada trascendental para estimular la evocación.

Pero ahora, ha nevado firme; con pertinencia esforzada, con agallas de rivalidad y propósito terco y baturro. En la nevada de la Purísima, después de aliviados los tejados de su carga inpoluta, que ineludiblemente iba a parar en la calle, formaba tan crecida barrera, que aislaba por completo a los transeúntes de opuestas aceras. No se veía siquiera el pico de los sombreros de los antípodas.

No hemos llegado a ese extremo, en esta nevada de 1926 y en los días de Navidad, pero... casi llegamos a la meta. Con un poquito más de esfuerzo, queda en ridículo la nevada por antonomasia, la de la Purísima.»

Si para las generaciones que vivieron la nevada de 1926 y las posteriores este evento es punto de referencia obligado con ocasión de precipitaciones de nieve, es interesante señalar que a finales del siglo pasado otra gran nevada la de 8 de diciembre de 1883 era el episodio de comparación respecto a aquélla, tenida incluso por más importante. Paradójicamente en la prensa local de 1883, en concreto la del día 11 de diciembre de $E l$ Serpis, son relativamente escasas las referencias a la nevada, considerada como un hecho ordinario: «El sábado por la mañana presentóse nuestra ciudad cubierta por una gruesa capa de blanca nieve, que fue aumentando con la que cayó durante casi todo el día. El

19 Ibidem, 15 de enero de 1980. 
fenómeno aunque puede considerarse ordinario, pues todos los años se repite, no dejó de causar extrañeza. La nieve caía impulsada por un fuerte viento que formaba ventisqueros, viéndose, por ello, amontonada en calles y tejados.»

Durante los diez días posteriores a esta nevada se siguen leyendo referencias a esta nevada similares en la temática a las aparecidas en 1926, incomunicación, medidas para despejar calles y tejados, alguna breve valoración, pero nada en comparación a la amplia información en cantidad y calidad de La Gaceta, y sin mención alguna a ruina económica y desastre industrial. No sabemos bien si la diferencia entre lo expresado en la prensa contemporánea y lo recordado en esta evocación se debe a una cuestión de percepción o al hecho de que por diversas razones fue menos espectacular en sus características e impactos la nevada de 1883 que la de 1926, pero el dato objetivo es que durante este siglo las evocaciones a la primera son mucho menos numerosas que las referidas a la segunda. Se puede intuir en este caso concreto un posible cambio en la repercusión periodística de los fenómenos atmosféricos excepcionales. Esta evolución en el mayor tratamiento de los fenómenos atmosféricos extraordinarios ha ido profundizándose a lo largo del presente siglo hasta desembocar en la consideración catastrofista que inunda las páginas de los diarios y las noticias de radio y televisión en nuestros días con ocasión de un episodio natural que perturba el funcionamiento de las sociedades. Se olvida, sin embargo, que la naturaleza tiene su propio comportamiento que el hombre debe esforzarse en conocer para evitar los efectos de episodios de este tipo en unas infraestructuras o actividades económicas que se han implantado, muy a menudo impropiamente, en territorios con ese natural comportamiento. Hay que recordar que el grado de riesgo frente a un hecho natural extraordinario no depende de la naturaleza - que está ahí y se debe conocer - sino del hombre que plasma territorialmente comportamientos poco acordes con el «natural» funcionamiento de aquélla.

Es frecuente escuchar en nuestros días que cada vez es más difícil ver nevar sobre la ciudad o la evocación de las nevadas de épocas pasadas, sobre todo la de 1926, por lo cuantiosa, o la de abril de 1958, por lo tardía, como algo difícil de repetir. No obstante, este tipo de evocaciones ya se hacían en fechas tan «lejanas» como 1926. En definitiva, encontramos frases muy similares a las que podemos leer hoy con motivo de episodios de estas características. Algo similar sucede con las lluvias, las sequías o las temperaturas. ¿Hay tantas diferencias entre el clima de principios de siglo o el siglo pasado y el de ahora como se nos quiere vender, o se trata sólo de una cuestión de percepción, en la que no falta la manida opinión de que «cualquier tiempo pasado fue mejor»?

La opinión de que nevadas y riadas destacables son hechos relevantes en sí mismos y puntos de referencia en la historia de los pueblos por lo que tienen de extraordinarios más aún que por el bien y mal que causan, no puede ser más acertada.

\section{Bibliografía}

GUALDA GÓMEZ, C.E. (1988): La Sierra de Mariola, Alicante, Universidad de Alicante, $268 \mathrm{pp}$.

KUNOW, P. (1966): El Clima de Valencia y Baleares, Valencia, Instituto Alfonso el Magnánimo, $239 \mathrm{pp}$.

OLCINA CANTOS, J. y MOLTÓ MANTERO, E. (1999): «La nevada de 1926. Repercusiones en la Montaña Alcoyana (Alicante)», Nimbus, $n^{\circ} 3$, Universidad de Almería, pp. 105-137. 
RUIZ URRESTARAZU, E. (1998): El clima del País Vasco a través de la prensa, Servicio Vasco de Meteorología, Grupo de Climatología de la Universidad del País Vasco, Vitoria, 212 pp.

\section{Fuentes documentales}

Ciudad de Alcoy, 1953-1999.

El Serpis, 1883.

Heraldo de Alcoy, 1914.

La Gaceta de Levante. Diario Independiente de Alcoy. 1926-1927. 